Rev. Adv. Mater. Sci. 57 (2018) 199-211

\title{
COMPARATIVE ANALYSIS OF DYNAMIC PLASTICITY MODELS
}

\author{
N.S. Selyutina ${ }^{1,2}$ and Yu.V. Petrov ${ }^{1,2}$ \\ ${ }^{1}$ Saint Petersburg State University, 199034, Universitetskaya nab. 7/9, St. Petersburg, Russia \\ ${ }^{2}$ Institute of Problems of Mechanical Engineering Russian Academy of Sciences (IPME RAS), \\ Extreme States Dynamics Department, V.O., 199178, Bolshoj pr., 61, St. Petersburg, Russia
}

Received: April 28, 2018

\begin{abstract}
In the paper, a new phenomenological interpretation of some of the principal temporal effects of high-rate plastic deformation of metals from a united viewpoint is represented. A comparative analysis of some of the well-known dynamic plastic deformation models is given. Influence of strain rate on the stress-strain relation in a wide range of strain rates for different types of aluminum alloys and steels is described by the relaxation model of plasticity, by original and improved empirical Johnson-Cook models, and by the phenomenological Rusinek-Klepaczko model. It is shown that the structural-time model ("the relaxation model of plasticity") is capable to effectively predict a wide spectrum of materials responses to fast and slow dynamic loading.
\end{abstract}

\section{INTRODUCTION}

Design of an efficient tool for calculations of stressstrain relationships in a wide range of strain rate is relevant for the development of engineering applications. Stress-strain relationships for metals under dynamic loading depend on the loading history. The simultaneous growth of the yield point and of the whole stress-strain curve after the beginning of yielding is typical evidence of the temporal nature of plastic deformation. However, experiments on soft steels [1-6] in a wide range of strain rates exhibit another temporal effect, known as a yield drop phenomenon. Often, the anomalous increase of the peak stress accompanied by a subsequent drop of stresses is ignored by many of existing models of dynamic plasticity. This is probably due to the fact that we obtain the same dependencies of yield stress on strain rate for each of temporal effect. It is necessary to develop approaches that would allow one to consider both temporal effects of strain rate sensitivity of the materials.
One of the most used approaches in computational fracture mechanics is an approach based on the Johnson-Cook model [11]. There are many extended models [7-10] developing Johnson-Cook model [11] in which better correspondence with experimental dependencies of the yield stress on strain rate than in previous improved models [12-17] is ensured by the use of the new empirical parameters. Some empirical parameters are the parameters principally associated with the specific strain rate. Thus, talking about independence of empirical parameters on strain rate is a big deal of the problem. The lack of physical meaning of empirical parameters leads to a limitation of the application of the model to the widest spectrum of materials responses to fast and slow dynamic loading.

The processes of plastic deformation are described by different phenomenological models [2029], which were developed for separate description of thermal and rate effects in metals. On a par with empirical dynamic plasticity models, rate effect, strain hardening and temperature sensitivity are si-

Corresponding author: N.S. Selyutina, e-mail: nina.selutina@gmail.com 
multaneously considered in the Rusinek-Klepaczko model $[18,19]$. Main advantages of the RusinekKlepaczko model and other constitutive models [3032] in comparison with empirical models are better correspondence with experimental results and a possibility of physical interpretation of effects observed in the yielding process. However, the main problem of all above mentioned models is the use of a large number of parameters that should be empirically determined.

In this paper, we consider the integral criterion of yielding based on the incubation time concept [33-37] as an approach to the yield strength value prediction at fixed strain rates, as well as more complex conditions of loading. The structural-temporal approach [38] was firstly proposed in fracture dynamics [39] and turned out to be a very effective tool to describe the temporal effects of crack growth initiation $[40,41]$. Generalization of the main idea of the incubation time concept for the case of plastic deformation phenomenon consists in consideration of the shear stress relaxation as a temporal process related to the defects motion $[42,43]$. The relaxation itself can be realized by various physical mechanisms depending on particular material. In frames of the incubation time approach we do not describe the relaxation mechanism explicitly, but do only the statement that it requires some characteristic time arising from the fact of the micro defects motion. In previous studies $[42,43]$ the relaxation model as a continuation of the structural-temporal approach to plasticity was formulated. The corresponding relaxation model of plasticity has three parameters; the first two of parameters are characteristic time and material sensitivity to the amplitude of local stress, the third one is the hardening parameter. Using these parameters, one can predict various stress-strain relationships for one material in a wide range of strain rates. So, the relaxation model of plasticity is proven to be an explanation of the dynamic effects of plasticity, for example appearance and disappearance of the yield drop phenomenon at strain rates $10^{-5}-10^{-2} \mathrm{~s}^{-1}$ [42] and $10^{2}-10^{3} \mathrm{~s}^{-1}[43]$.

Here we compare some of the well-known dynamic plastic deformation models by prediction of temporal effects of yielding process. We will give expressions for some of the parameters of empirical models and of the extended Rusinek-Klepaczko model through characteristics of the relaxation model of plasticity. Strain rate dependencies of yield stresses for DP 1200 steel, DP 1400 steel and 6082T6 aluminium alloys by the incubation time criterion, the Cowper-Symonds model and the Johnson-
Cook model are analyzed. Stress-strain dependencies in a wide range of strain rate for AA 7075 T6, AA2519A aluminium alloys and B500A, AISI 4340, TRIP800, 2.3Ni-Cr, $590 \mathrm{MPa}$ grade steels will be predicted by our phenomenological model, by existing original and improved empirical models (the Johnson-Cook model, the Zerilli-Armstrong model, the Khan-Huang-Liang model) and by another the extended phenomenological model by RusinekKlepaczko. Main issues of existing dynamic plasticity models in simultaneous prediction of monotonic growth of yield stress and yield drop effect at small strain will be discussed. It will be shown that parameters of original and modified empirical models and the Rusinek-Klepaczko model are not invariant to the loading history.

\section{INCUBATION TIME CRITERION OF YIELDING}

To model temporal effects of plastic deformation of the materials, we consider a structural-temporal approach based on the idea of material incubation time. Yield condition in the material for an arbitrary pulse of loading by the structural-temporal approach or the incubation time criterion of yielding (IT criterion) $[33,34,37]$ is determined by the inequality:

$$
\frac{1}{\tau} \int_{t-\tau}^{t}\left(\frac{\Sigma(s)}{\sigma_{y}}\right)^{\alpha} \mathrm{d} s \leq 1,
$$

where, $\Sigma(t)$ is a function describing the time dependence of stress, $\tau$ is the incubation time (or characteristic relaxation time), $\sigma_{y}$ is the static yield stress, $\alpha$ is a parameter of amplitude sensitivity of the material. The time of the beginning of the macroscopic yield is defined as the moment at which the equality sign in Eq. (1) is attained. The introduced time parameter $\tau$, independent of the specific features of deformation and sample geometry, makes it possible to predict the behaviour of the yield strength of materials under static and dynamic loads [33]. It was shown in the paper [43] that the incubation time can be related to different physical mechanisms of plastic deformation. It is assumed that the incubation time parameter can take different values depending on initial defective structures of the material. In other words, two samples, made of the same material by means of two different structures prior to the beginning of the plastic strain tests, are considered as samples made of two different materials. Thus, two independent parameters of the IT criterion of $\tau$ and $\alpha$ characterize the rate (temporal) 
and amplitude sensitivity of the material, respectively.

Let us formulate the simplest way of modeling of strain rate dependence of the yield stress based on the IT criterion (1). Consider the linear elastic deformation law $\Sigma(t)=E \dot{\varepsilon} t H(t)$, where $E$ is the Young's modulus and $\dot{\varepsilon}$ is the constant strain rate under load, $H(t)$ is the Heaviside step function. Having written the left-hand side of (1) under the condition that the yield starts at time $t_{y}$, one can express the dynamic yield stress $\Sigma d=\Sigma\left(t_{y}\right)$ in terms of the strain rate of material:

$$
\Sigma_{d}(\dot{\varepsilon})= \begin{cases}{\left[(\alpha+1)\left(\sigma_{y}\right)^{\alpha} E \dot{\varepsilon} \tau\right]^{1 /(\alpha+1)},} & \dot{\varepsilon} \geq \frac{(\alpha+1)^{1 / \alpha} \sigma_{y}}{E \tau} ; \\ \sigma_{y}+\left(1-\frac{1}{(\alpha+1)^{1 / \alpha}}\right) E \dot{\varepsilon} \tau, & \dot{\varepsilon}<\frac{(\alpha+1)^{1 / \alpha} \sigma_{y}}{E \tau} .\end{cases}
$$

Substituting temperature dependence of static yield stress [44] and parameters of the IT criterion [36], the strain rate dependence of yield stress in Eq. (2) as a function of temperature can be derived. As one can see, Eq. (2) gives a simple explicit description of rate dependence of the yield stress in a wide range of strain rates. Note that the behaviour of the particular material under different temperatures will be characterized by its own incubation time and amplitude sensitivity parameter. These characteristics of the IT criterion create a set of material parameters invariant to the strain rate. This fact of invariance of parameters of the IT criterion to loading history and physical mechanisms of plasticity would allow one to compare two materials in a wide range of strain rates including of slow (quasi-static) and fast loading. To establish a dominant range of strain rates, where the yield stress of one material is greater than that for another one, we analyze their characteristic times $[36,45]$. The material with a bigger characteristic time may possess the greater yield strength at slow loading, whereas its yield stress at fast loading may decrease. This temporal effect, known as an inversion of yield strengths for two materials with different temperatures [36] or different of grain sizes [45] was observed in a wide range of strain rates and effectively predicted by the IT criterion.

\section{DEFINITION OF YIELD POINT IN NUMERICAL MODELS}

Let us consider strain rate dependencies of the yield stress following from the Cowper-Symonds model [46] and the Johnson-Cook model [29] and compare them with conclusions driven from the IT criterion. Simple empirical models $[29,46]$ of high-rate deformation metals were initially developed for the case of large deformations. One of the first empirical models in this field was the Cowper-Symonds model (CS model) [46] that can be written in the following form:

$$
\Sigma_{c S}(\dot{\varepsilon})=\sigma_{y}\left(1+\left\{\frac{\dot{\varepsilon}}{B_{C S}}\right\}^{1 / a_{c s}}\right) .
$$

where $B_{C S}$ and $q_{C S}$ are two empirical parameters of Cowper-Symonds model. On the basis of the original Johnson-Cook model (JC model) [29] we write the dynamic yield stress as a Proposed power function of strain rate in Eq. (3) is often used in improved models of original Johnson-Cook model (JC model) [29]. On the other hand, according to the $\mathrm{JC}$ model, the equation for the dynamic yield stress can be defined as:

$$
\Sigma_{J C}\left(\varepsilon_{p}\right)=\left(A_{J C}+B_{J C} \varepsilon_{p}^{n_{J C}}\right)\left(1+C_{J C} \ln \left\{\frac{\dot{\varepsilon}}{\dot{\varepsilon}_{J C}}\right\}\right)\left(1-\left\{\frac{T-T_{0}}{T_{m}-T_{0}}\right\}^{m_{T}}\right),
$$

where $A_{J C}, B_{J C}, n_{J C}$ are parameters of the empirical hardening law; $C_{J C}, \dot{\varepsilon}_{J C}$ are parameters of rate dependence of yield stress; $T, T_{0}, T_{m}, m_{T}$ are parameters of temperature dependence of yield stress. In order to predict the dependence of yield stress on strain rate at fixed temperatures, both the CS model and the JC model require two material parameters.

Let us compare theoretical dependencies of the yield stress on strain rate, calculated by the CS model, the $\mathrm{JC}$ model and the IT criterion. Using experimental results of tension in split Hopkinson bar tests [47] of DP1200 steel $\left(\sigma_{y}=1.1 \mathrm{GPa} ; E=210 \mathrm{GPa}\right)$ and DP 1400 steel $\left(\sigma_{y}=1.3 \mathrm{GPa} ; E=210 \mathrm{GPa}\right)$ we examine 


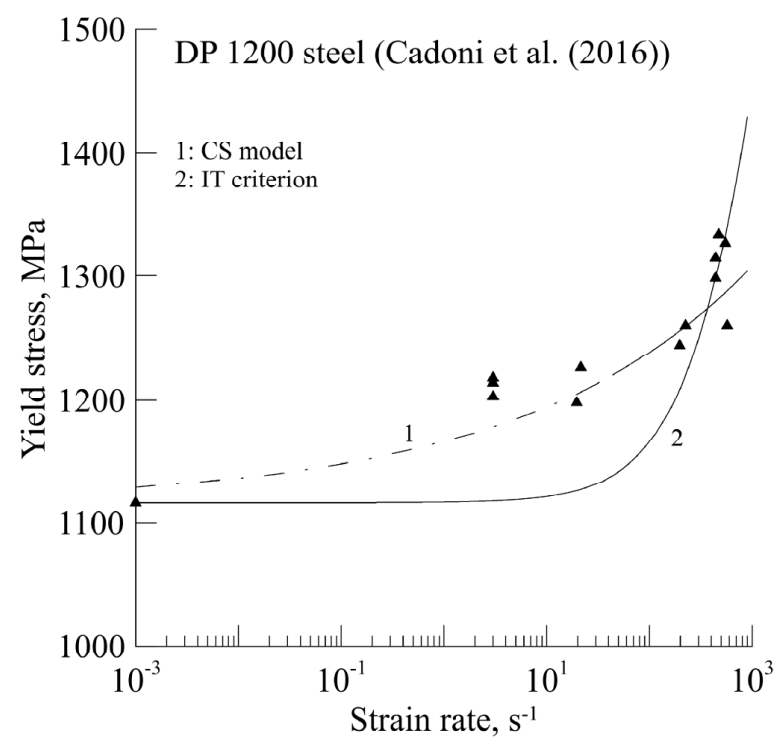

Fig. 1. Rate dependence of yield stress of DP1200 steel (data from [47]), plotted on CS model (1 line) and IT criterion (2 line).

efficiencies of the CS model and the IT criterion (Figs. 1 and 2). Fitted in [47] parameters of the CS model and parameters of the IT criterion for steels are presented in Table 1. Both models give a good correspondence with experimental data for steels in the range of strain rate $10^{-3}-10^{3} \mathrm{~s}^{-1}$. An intersection of theoretical rate dependencies, plotted by the CS model and the IT criterion, are observed at strain rate, and occurs at approximately $10^{3} \mathrm{~s}^{-1}$. Experi-

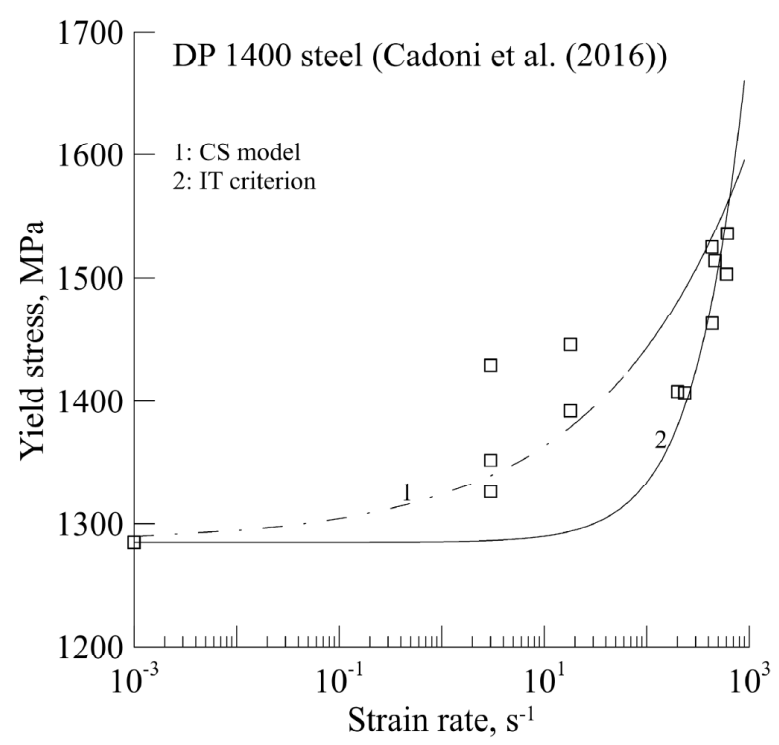

Fig. 2. Rate dependence of yield stress of DP1400 steel (data from [47]), plotted on CS model (1 line) and IT criterion (2 line).

mental results in split Hopkinson bar tests for $\mathrm{Al}$ alloy 6082-T6 ( $\sigma_{y}=335 \mathrm{MPa}$; $E=71.7 \mathrm{GPa}$ ) [48], and theoretical dependencies of yield stress on strain rate are plotted in Fig. 2. Table 2 shows parameters of the $\mathrm{JC}$ model, obtained in the paper [48], and parameters of the IT criterion for Al alloy 6082-T6. As shown in Fig. 3, the JC model gives a satisfactory estimation of the yield point only up to a strain rate of the order of $10^{4} \mathrm{~s}^{-1}$. In an opposite case, the

Table 1. Definition of parameters of CS model by IT criterion and comparison these parameters with original values.

\begin{tabular}{llllllll}
\hline Material & \multicolumn{2}{l}{ IT criterion } & \multicolumn{2}{l}{ CS model } & \multicolumn{3}{l}{ IT criterion by Eqs. (5) } \\
& $\alpha$ & $\tau, \mu \mathrm{s}$ & $B_{C S}, \mathrm{~s}^{-1}$ & $q_{C S}$ & $B_{C S}^{s t}\left(\dot{\varepsilon}, \mathrm{s}^{-1}\right), \mathrm{s}^{-1}$ & $B_{C S}^{d}, \mathrm{~s}^{-1}$ & $q_{C S}$ \\
\hline Al alloy 7449-T7651 [51] & 1 & 0.94 & 15007 & 0.95 & $15080(16500)$ & 3944 & 2 \\
B500A steel [6] & 1 & 1.48 & 141917 & 2.896 & $141200(92)$ & 901 & 2 \\
DP 1200 steel [47] & 4.14 & 3.78 & 8570000 & 5.14 & $8708000(390)$ & 339.6 & 5.14 \\
DP 1400 steel [47] & 2.27 & 4 & 94000 & 3.27 & $94320(910)$ & 339.6 & 3.27 \\
\hline
\end{tabular}

Table 2. Definition of parameters of JC model by IT criterion and comparison these parameters with original values.

\begin{tabular}{llllll}
\hline Material & IT criterion & & JC model & & $\begin{array}{l}\text { IT criterion by } \\
\text { Eq. }(6) \\
\end{array}$ \\
& $\alpha$ & $\tau, \mu \mathrm{s}$ & $\dot{\varepsilon}_{J C}, \mathrm{~s}^{-1}$ & $C_{J C}$ & $\mathrm{C}_{J c}(\dot{\varepsilon})$ \\
\hline Al alloy 7075-T6 [52] & 1 & 6.5 & 0.001 & 0.033 & $0.036\left(1000 \mathrm{~s}^{-1}\right)$ \\
Al alloy 6082-T6 [48] & 1 & 0.27 & 0.001 & 0.00519 & $0.00514\left(2600 \mathrm{~s}^{-1}\right)$ \\
ASTM A36 steel [50] & 26 & 9.58 & 1 & 0.0017 & $0.00186\left(10 \mathrm{~s}^{-1}\right)$ \\
Ni [16] & 1 & 0.5 & 1 & 0.01 & $0.12\left(300 \mathrm{~s}^{-1}\right)$ \\
\hline
\end{tabular}




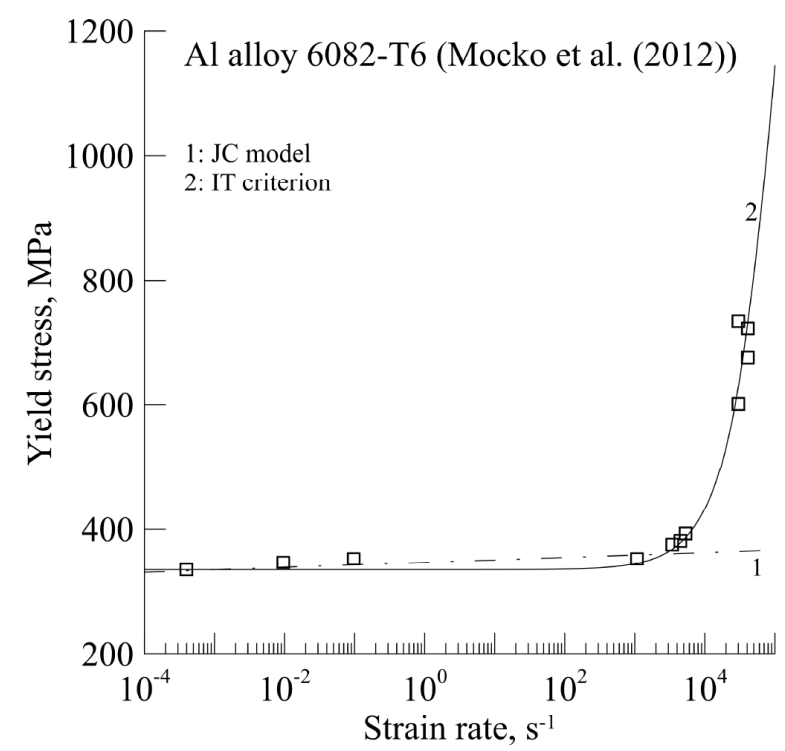

Fig. 3. Rate dependence of yield stress of Al alloy 6082-T6 (data from [48]), plotted on JC model (1 line) and IT criterion (2 line).

IT criterion allows predicting values of the yield point for a whole range of strain rates $10^{-4}-10^{5} \mathrm{~s}^{-1}$, including experimental data of yield stress for strain rates greater than $10^{4} \mathrm{~s}^{-1}$.

The IT criterion allows us to evaluate the parameters of the CS model and the $\mathrm{JC}$ model. In the papers $[44,49]$, expressions for parameters of the $\mathrm{JC}$ model and the CS model were derived:

$$
\begin{aligned}
& q_{C S}=\alpha+1 ; \\
& B_{c S}^{s t}(\dot{\varepsilon})=\left[\left(1-\frac{1}{(\alpha+1)^{1 / \alpha}}\right) \frac{E_{\tau}}{\sigma_{y}}\right]^{-\alpha-1} \dot{\varepsilon}^{-\alpha} ; \\
& B_{c S}^{d}=\frac{1}{\alpha+1} \frac{\sigma_{y}}{E \tau} \\
& C_{J C}\left(\dot{\varepsilon}, \dot{\varepsilon}_{J c}\right)=\left(1-\frac{1}{(\alpha+1)^{1 / \alpha}}\right) \frac{E \dot{\varepsilon} \tau}{\sigma_{y}}\left[\ln \left(\frac{\dot{\varepsilon}}{\dot{\varepsilon}_{J C}}\right)\right]^{-1} .
\end{aligned}
$$

One of two simple empirical parameters is associated only with the parameter of the amplitude sensitivity of the material $(\alpha)$, and the other, with two parameters of the incubation time criterion $(\alpha, \tau)$. Using this interpretation, one of the parameters of the CS model $\left(B_{C S}\right)$ and of the JC model $\left(C_{J C}\right)$ is a function of strain rate. Moreover, the parameter $\left(C_{J C}\right)$ also depends on the strain rate $\left(\dot{\varepsilon}_{J C}\right)$, which takes on different meanings $\left(0.001 \mathrm{~s}^{-1}, 1 \mathrm{~s}^{-1}\right)$ depending on the material as shown in Table 1. Thus, parameters of empirical models reveal the dependence on strain rate.
Table 1 and Table 2 represent empirical parameters of the $\mathrm{JC}$ model and the CS model for different metals (nickel; steels: DP 1200 [47], DP 1400 [47], ASTM A36 [50], B500A [6]; aluminum alloys: 7449T7651 [51], 7075-T6 [52], 6082-T6 [48]) which we can establish two parameters of the IT criterion, calculated here and in the paper [49]. These parameters correspond to original parameters of the $\mathrm{JC}$ model and the CS model. So, parameters $B_{c S}^{s t}(\dot{\varepsilon})$ and $C_{J C}(\dot{\varepsilon})$ depend on different values of strain rates (see calculated parameters $B_{c S}^{s t}(\dot{\varepsilon})$ and $C_{J C}(\dot{\varepsilon})$ ). Thus, three parameters are actually used for prediction of theoretical rate dependencies of yield stress by the CS model and the JC model. Modifications of simple empirical models for prediction stress-strain relationships under dynamic loading proposed in [10] do strongly complicate the original empirical model by putting into consideration a number of new parameters. However, the IT criterion uses only two invariant to the history of loading characteristics, which do not require further modifications at high strain rates.

\section{THE RELAXATION MODEL OF PLASTICITY}

Many principal temporal effects of yielding influencing stress-strain relations with increase of the strain rate are taken into account by the incubation time concept. To construct the whole set of stress-strain curves in a wide range of strain rates, we proposed the generalized structural-temporal approach to plastic deformation, known as the relaxation model of plasticity (RP model) [42,43].

Let us introduce a dimensionless relaxation function $0<\gamma(t) \leq 1$, defined as follows

$$
\gamma(t)=\left\{\begin{array}{l}
1, \frac{1}{\tau} \int_{t-\tau}^{t}\left(\frac{\Sigma(s)}{\sigma_{y}}\right)^{\alpha} \mathrm{d} s \leq 1, \\
\left(\frac{1}{\tau} \int_{t-\tau}^{t}\left(\frac{\Sigma(s)}{\sigma_{y}}\right)^{\alpha} \mathrm{d} s\right)^{-1 / \alpha}, \\
\frac{1}{\tau} \int_{t-\tau}^{t}\left(\frac{\Sigma(s)}{\sigma_{y}}\right)^{\alpha} \mathrm{d} s>1 .
\end{array}\right.
$$

Equality $\gamma(t)=1$ in (7) corresponds to the case of purely elastic deformation. Gradual decrease of the relaxation function in the range $0<\gamma(t)<1$ describes a transition to the plastic deformation stage. During the plastic stage of deformation, $t \geq t_{y}$, the relaxation function satisfies the condition 


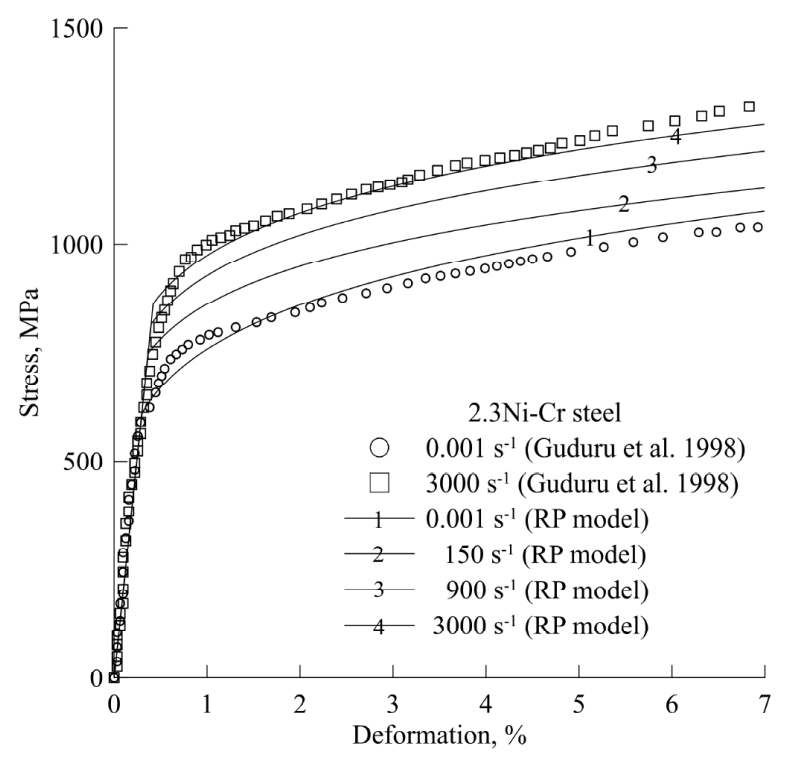

Fig. 4. Stress-strain dependences of $2.3 \mathrm{Ni}-\mathrm{Cr}$ steel (data from [54]) on strain rates $0.001 \mathrm{~s}^{-1}$ and 2650 $\mathrm{s}^{-1}$, predicted by relaxation model ( $E=205 \mathrm{GPa}$, $\left.\sigma_{y}=610 \mathrm{MPa} ; \alpha=20, \tau=70 \mu \mathrm{s}, \beta=0.18\right)$.

$$
\frac{1}{\tau} \int_{t-\tau}^{t}\left(\frac{\gamma(t) \Sigma(s)}{\sigma_{y}}\right)^{\alpha} d s=1
$$

The actual stress $\sigma(t)$ in a deformed specimen is equal to $\sigma(t)=E \gamma^{1-\beta}(t) \varepsilon(t)$ where $\beta$ is a scalar parameter $(0 \leq \beta \leq 1)$ characterizing the level of hardening; case $\beta=0$ corresponds to the plastic deformation without stain hardening. Considering the stages of elastic and plastic deformations separately, we can obtain from (8) the following stress-strain relation in the case of a linear growth of strain $\varepsilon(t)=\dot{\varepsilon} t H(t)$ (the constant strain rate)

$$
\sigma(\varepsilon(t))= \begin{cases}E \varepsilon(t), & \varepsilon(t) / \dot{\varepsilon}<t_{*} \\ E \gamma^{1-\beta}(\varepsilon(t) / \dot{\varepsilon}) \varepsilon(t), & \varepsilon(t) / \dot{\varepsilon} \geq t_{*}\end{cases}
$$

where we take into account that $t=\varepsilon(t) / \dot{\varepsilon}$.

We see that the parameter $\beta$ effects on the relaxation function $\gamma(t)$. In initial formulation of the relaxation model of plasticity [42,53], acceptable region of the parameter $\beta$ is from 0 to 1 .

All parameters $\alpha, \tau, \beta$ are invariant to loading history and only depend on structural transformations in the material. Assessments of these parameters allow predicting different types of stress-strain dependences for the same material in a wide range of strain rates.

In order to determine a dependence of stress on plastic strains (without elastic part of deformation) by the RP model, one can rewrite:
$\sigma\left(\varepsilon_{p}(t)\right)=E \gamma^{1-\beta}\left(\varepsilon_{p}(t) / \dot{\varepsilon}\right) \varepsilon_{p}(t)$,

where $\varepsilon_{p}(t)=\varepsilon(t)-\dot{\varepsilon} t . H\left(t_{t}\right)$ is plastic strain.

It is interesting to analyze stress-strain relations in the range of strain rate $10^{-3}-10^{3} \mathrm{~s}^{-1}$ under $\mathrm{dy}$ namic and quasi-static loading in tensile $[2,5]$ and compression [54] experiments studying the yield drop phenomenon. Theoretical stress-strain dependencies, plotted in Figs. 4- 6 by the RP model, for 2.3 $\mathrm{Ni}-\mathrm{Cr}$ steel $\left(E=205 \mathrm{GPa}, \sigma_{y}=610 \mathrm{MPa}\right.$ ), TRIP 800

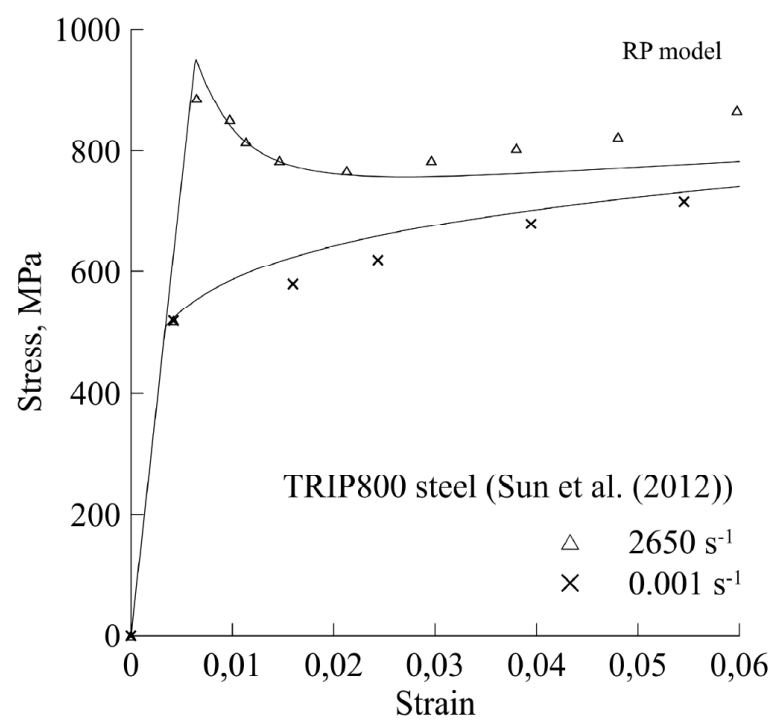

Fig. 5. Stress-strain dependences of TRIP800 steel (data from [5]) on strain rates $0.001 \mathrm{~s}^{-1}$ and 2650 $\mathrm{s}^{-1}$, predicted by relaxation model of plasticity $\left(E=150 \mathrm{GPa}, \sigma_{y}=510 \mathrm{MPa} ; \alpha=2, \tau=2.8 \mu \mathrm{s}, \beta=0.13\right.$ ).

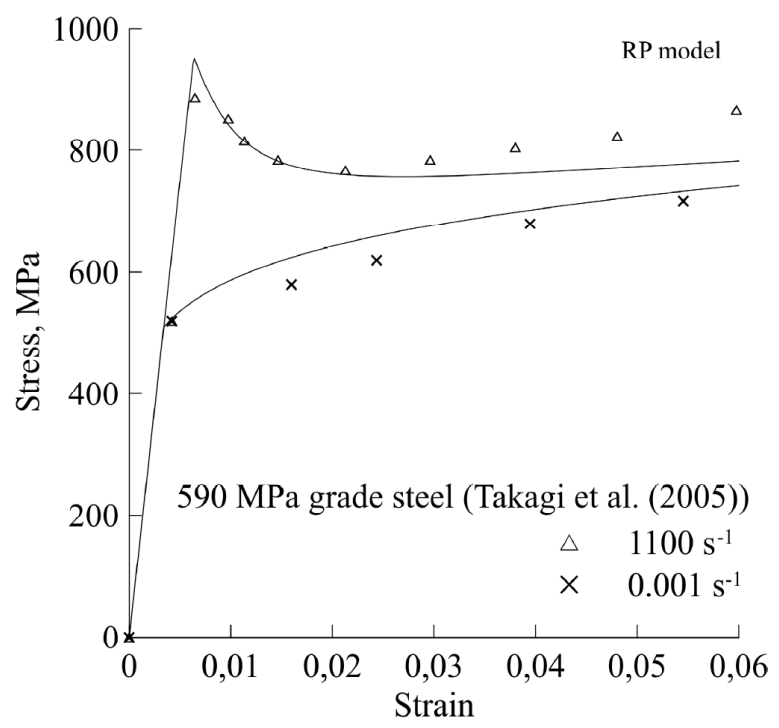

Fig. 6. Stress-strain dependences of $590 \mathrm{MPa}$ grade steel (data from [2]) on strain rates $0.001 \mathrm{~s}^{-1}$ and $1100 \mathrm{~s}^{-1}$, predicted by relaxation model of plasticity $\left(E=210 \mathrm{GPa}, \sigma_{y}=280 \mathrm{MPa} ; \alpha=2.5, \tau=8.5 \mu \mathrm{s}\right.$, $\beta=0.17)$. 
steel $\left(E=150 \mathrm{GPa}, \sigma_{y}=510 \mathrm{MPa}\right), 590 \mathrm{MPa}$ grade steel $\left(E=210 \mathrm{GPa}, \sigma_{y}=280 \mathrm{MPa}\right.$ ) gives a good correspondence with experimental data. The RP model parameters equal to $\alpha=20, \tau=70 \mu \mathrm{s}, \beta=0.18$ for 2.3 Ni-Cr steel, $\alpha=2, \tau=2.8 \mu \mathrm{s}, \beta=0.13$ for TRIP 800 steel, $\alpha=2.5, \tau=8.5 \mu \mathrm{s}, \beta=0.17$ for $590 \mathrm{MPa}$ grade steel. Stress-strain dependencies of all metals at quasistatic loading $\left(\dot{\varepsilon}=0.001 \mathrm{~s}^{-1}\right)$ and dynamic stressstrain curves of $2.3 \mathrm{Ni}-\mathrm{Cr}$ steel with increase of strain rate (Fig. 4) have a smooth transition to the initial stage of plastic deformation. Anomalous effects of yield stresses greater than normal stress at small deformation and subsequent relaxation process for TRIP 800 steel (Fig. 5) and 590 MPa grade steel (Fig. 6) at strain rate more than $10^{3} \mathrm{~s}^{-1}$ are observed.

The RP model allows one predicting a wide spectrum of stress-strain relations in conditions of quasistatic and dynamic loading, which can be grouped into two types. The first type includes typical stressstrain relations where the yield stress changes proportionally with the whole deformation curve (Fig. 4). Appearance and disappearance of the yield drop phenomenon, observed in Figs. 5 and 6 imply the other type of stress-strain curves.

\section{TEMPORAL EFFECTS OF YIELDING}

Dynamic effect of yield drop phenomenon at early stages of deformation, arising from the increase of strain rate, is a reflection of presence of typical relaxation processes, which in general can be represented by characteristic times. Note that in the case the yield drop, the yield process can be defined by two critical stresses called "high yield stress" and "low yield stress". Yield drop effect is crucial for the understanding of the dynamic nature of yielding. By the proposed the RP model, the peak stress or high yield stress defines the upper yield point. It is shown further that dynamic effect of yield drop arising from the increase of strain rate is not predicted by existing empirical models and the phenomenological Rusinek-Klepaczko model.

\subsection{Modifications of the $\mathrm{JC}$ model}

Empirical models of plastic deformation are not always capable to describe temporal effects of yielding in the material. Let us consider different extensions of the original the JC model (4). In the paper [55], the modification of hardening part of the original JC model, known as the Liu-He-Chen-Tang model (LHCT model), can be written as:
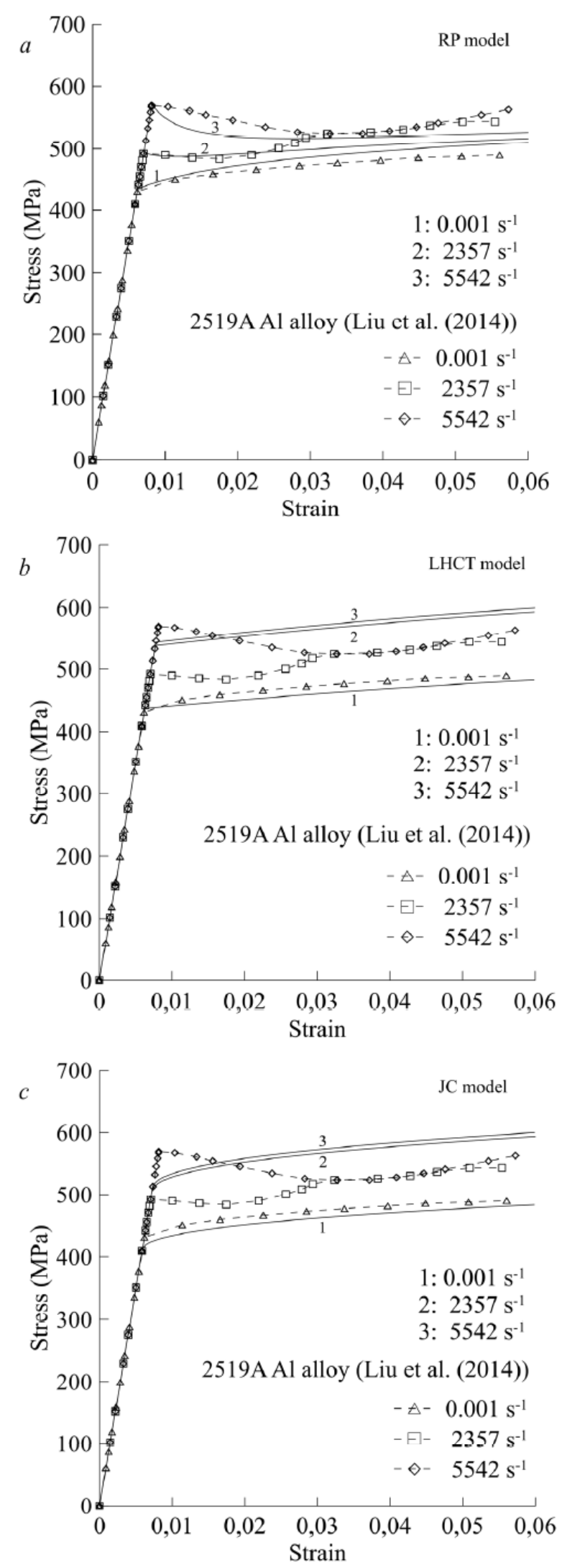

Fig. 7. Stress-strain relationships of aluminum alloy 2519 A (data from [55]), plotted by RP model (a), LHCT model (b) and JC model (c). 


$$
\begin{aligned}
& \Sigma_{M J C 2}\left(\varepsilon_{p}\right)=A_{J C}\left(1+E_{M J C 2} \varepsilon_{p}+F_{M J C 2} \varepsilon_{p}^{2}\right) \times \\
& \left(1+C_{J C} \ln \left\{\frac{\dot{\varepsilon}}{\dot{\varepsilon}_{J C}}\right\}\right)\left(1-\left\{\frac{T-T_{0}}{T_{m}-T_{0}}\right\}^{m_{\tau}}\right),
\end{aligned}
$$

where $E_{\mathrm{M} J C_{2}}, F_{\mathrm{M} J C_{2}}$ are new empirical parameters. Comparison of theoretical stress-strain relationships of 2519A aluminium alloy [55] for strain rate of 0.001 $\mathrm{s}^{-1}, 2357 \mathrm{~s}^{-1}, 5542 \mathrm{~s}^{-1}$, plotted before strain $6 \%$ by the JC model at $\dot{\varepsilon}_{J C}=1 \mathrm{~s}^{-1}$, the LHCT model at $\dot{\varepsilon}_{J C}=1 \mathrm{~S}^{-1}$ and the RP model, are presented in Fig. 7. Both the JC model and the LHCT model produce theoretical monotonic increase of the yield point with strain rate on the initial stage of plastic deformation and demonstrate the absence of the yield drop that does not correspond to experimental data [55]. On the contrary the RP model gives proper yield drop effect.

In other the Huh-Kang model (HK model) [56] a component of strain rate sensitivity of the yield stress (presented in the $\mathrm{JC}$ model) is significantly modified by the following form:

$$
\begin{aligned}
& \Sigma_{J C}\left(\varepsilon_{p}\right)=\left(A_{J C}+B_{J C} \varepsilon_{p}^{n_{J C}}\right)\left(1+C_{J C} \ln \left\{\frac{\dot{\varepsilon}}{\dot{\varepsilon}_{J C}}\right\}+\right. \\
& \left.C_{H K}\left(\ln \left\{\frac{\dot{\varepsilon}}{\dot{\varepsilon}_{J C}}\right\}\right)^{2}\right)\left(1-\left\{\frac{T-T_{0}}{T_{m}-T_{0}}\right\}^{m_{r}}\right),
\end{aligned}
$$

where $C_{\mathrm{HK}}$ is the empirical parameter. Given the decisive contribution to the rate sensitivity this parameter, the parameter $C_{\mathrm{HK}}$ can be defined through parameters of the IT criterion:

$$
C_{H K}=\left[(\alpha+1) \sigma_{y} E \dot{\varepsilon} \tau\right]^{1 /(\alpha+1)}\left[\ln \left\{\frac{\dot{\varepsilon}}{\dot{\varepsilon}_{J C}}\right\}^{2}\right]^{-1} .
$$

A good correspondence of dependencies of stress on plastic strain under static $\left(0.001 \mathrm{~s}^{-1}\right)$ and dynamic loading $\left(1740 \mathrm{~s}^{-1}\right)$ for entire compared models (the HK model, the JC model and the RP model) with experimental data AISI4340 steel [57] is observed in Fig. 8. Both the HK model (Fig. 8b) and the $\mathrm{JC}$ model (Fig. 8c) are identical behave before strain $5 \%$ in spite of use in the HK model of new parameters.

In the paper [49], the Couque model [16] gives a good correspondence of strain rate dependence of yield stress for nickel unlike the JC model:

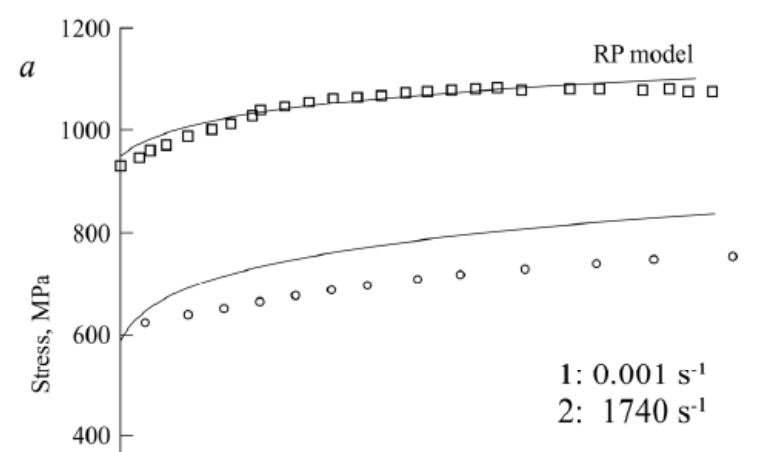

AISI 4340 steel (Tuazon et al. (2014))
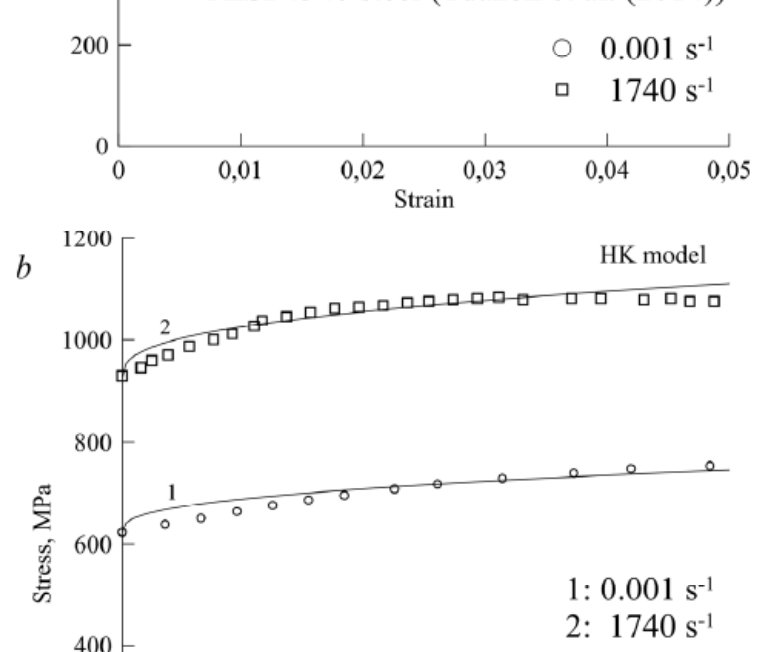

AISI 4340 steel (Tuazon et al. (2014))
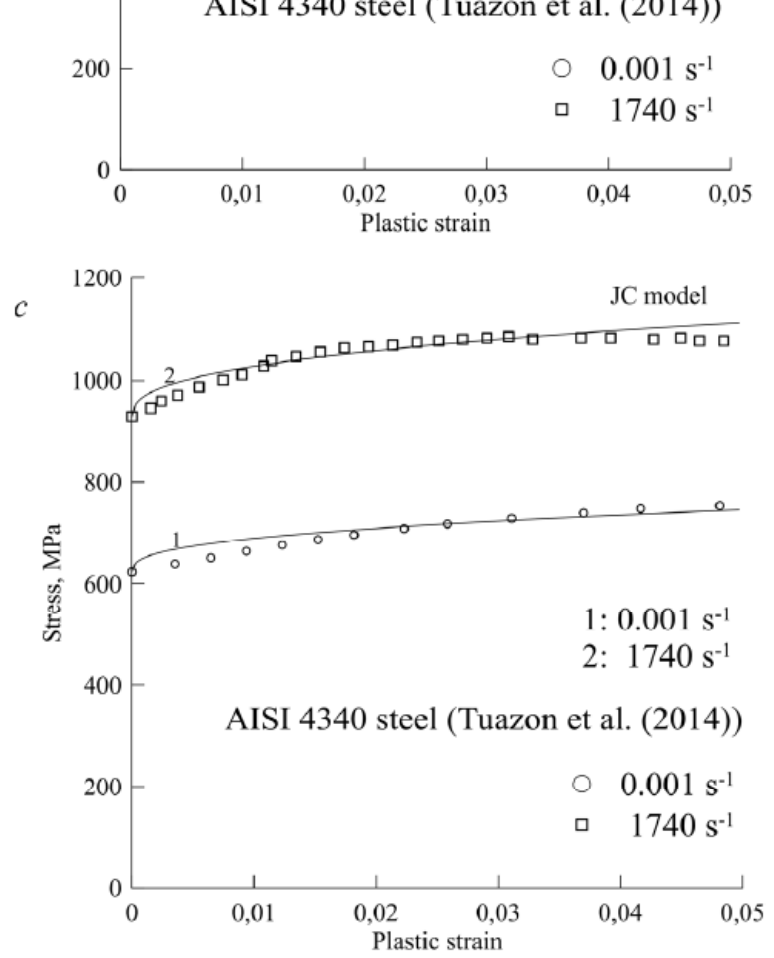

Fig. 8. Stress-strain relationships of AISI4340 steel (data from [57]), plotted by RP model (a), HK model (b) and JC model (c). 
$\Sigma_{M J C}\left(\varepsilon_{p}\right)=\left(A_{J C}+B_{J C} \varepsilon_{p}^{n_{J C}}\right)\left(1+C_{J C} \ln \left\{\frac{\dot{\varepsilon}}{\dot{\varepsilon}_{J C}}\right\}+D_{M J C}\left\{\frac{\dot{\varepsilon}}{\dot{\varepsilon}_{M J C}}\right\}^{k_{M C}}\right)\left(1-\left\{\frac{T-T_{0}}{T_{m}-T_{0}}\right\}^{m_{T}}\right)$,

where two new empirical parameters of $D_{M J C}, k_{M J C}$ can be identified by the IT criterion:

$$
D_{M J C}=\left((\alpha+1) \frac{E \dot{\varepsilon}_{M J C} \tau_{y}}{\sigma_{y}}\right)^{1 /(\alpha+1)}, k_{M J C}=\frac{1}{\alpha+1} .
$$

Recently, extended empirical models are formulated using combined functions of temperature, strain and strain rate, such as the Khan-Huang-Liang (KHL) model [58]:

$$
\Sigma_{K H L}\left(\varepsilon_{p}\right)=\left(A_{K H L}+B_{K H L}\left(1-\frac{\ln \dot{\varepsilon}}{\ln D_{K H L}}\right)^{k_{k H L}} \varepsilon_{p}^{n_{K H L}}\right)\left(\frac{\dot{\varepsilon}}{\dot{\varepsilon}_{J C}}\right)^{c_{K H H}}\left(1-\left\{\frac{T-T_{0}}{T_{m}-T_{0}}\right\}^{m_{T}}\right),
$$

where $A_{K H L}, B_{K H L}, C_{K H L}, D_{K H L}, k_{K H L}, n_{K H L}, \dot{\varepsilon}_{K H L}$ are empirical parameters, and integrated model [59] based on the JC model, the Zerilli-Armstrong model [29], written in Eq. (17):

$$
\begin{aligned}
& \Sigma_{\mathrm{zA}}=\left\{\begin{array}{l}
C_{0}^{B C C}+C_{1}^{B C C} \varepsilon^{n_{z A}} \exp \left(-C_{3}^{B C C} T+C_{4}^{B C C} T \ln \left\{\frac{\dot{\varepsilon}}{\dot{\varepsilon}_{J C}}\right\}\right)+C_{5}^{B C C} \varepsilon^{n_{z A}} \\
C_{0}^{F C C}+C_{1}^{F C C} \varepsilon^{n_{z A}} \exp \left(-C_{3}^{F C C} T+C_{4}^{F C C} T \ln \left\{\frac{\dot{\varepsilon}}{\dot{\varepsilon}_{J C}}\right\}\right)
\end{array},\right. \\
& \Sigma_{J Z K}=\left(\sigma_{y}+B_{K H L}\left(1-\frac{\ln \dot{\varepsilon}}{\ln D_{K H L}}\right)^{n_{1}}\left(\varepsilon_{p}\right)^{n_{0}}\right) \exp \left[-\left(C_{3}+C_{4} \varepsilon\right)\left\{\frac{T-T_{0}}{T_{m}-T_{0}}\right\}^{m_{T}}+\left(C_{5}+C_{6}\left\{\frac{T-T_{0}}{T_{m}-T_{0}}\right\}^{m_{T}}\right) \ln \left\{\frac{\dot{\varepsilon}}{\dot{\varepsilon}_{J C}}\right\}\right],
\end{aligned}
$$

where $C_{0}{ }^{B C C}, C_{1}{ }^{B C C}, C_{2}{ }^{B C C}, C_{3}{ }^{B C C}, C_{4}{ }^{B C C}, C_{5}{ }^{B C C}, n_{\mathrm{ZA}}, C_{0}{ }^{F C C}, C_{1}{ }^{F C C}, C_{3}{ }^{F C C}, C_{4}{ }^{F C C}$ are empirical parameters of the Zerilli-Armstrong model for BCC and FCC metals; $n_{0}, n_{1}, C_{3}, C_{4}, C_{5}, C_{6}$ are other empirical parameters.

Figs. 7 and 8 indicate that extended models based on the $\mathrm{JC}$ model cannot improve the prediction of stress-strain relationships with increasing strain rates. On the one hand, the IT criterion and the CS model require only two parameters for prediction the strain rate sensitivity of yield stress. On the other hand, more than two parameters are used in extended the HK model and the Couque model. Thus, a set of parameters in extended empirical models (Table 3 ) for description of dependence of yield stress on strain rate is often insufficient (for example, even seven parameters of the KHL model). Extended empirical models have many parameters (Table 3), but they do not provide one a unified point of view on predicting the temporal effects of plastic deformation.

Table 3. Empirical models based on original Johnson-Cook model.

\begin{tabular}{llll}
\hline Model & Reference & Strain rate parameters & Hardening parameters \\
\hline JC model & {$[29]$} & $C_{J C}, \dot{\varepsilon}_{J C}$ & $B_{J C}, n_{J C}$ \\
CS model & {$[46]$} & $B_{C S}, q_{C}$ & - \\
LHCT model & {$[55]$} & $C_{J C}, \dot{\varepsilon}_{J C}$ & $E_{M J C}, F_{M J C}$ \\
HK model & {$[56]$} & $C_{J C}, \dot{\varepsilon}_{J C}, C_{H K}$ & $B_{J C}, n_{J C}$ \\
Couque model & {$[16]$} & $C_{J C}, \dot{\varepsilon}_{J C}, D_{M J K}, k_{M J K}$, & $B_{J C}, n_{J C}$ \\
KHL model & {$[58]$} & $A_{K H L}, B_{K H L}, C_{K H L}, D_{K H L}, k_{K H L}, n_{K H L}, \dot{\varepsilon}_{J C}$, \\
\hline
\end{tabular}




\subsection{The extended Rusinek-Klepaczko model, the RP model and the JC model}

In order to describe the plastic flow of mild steel, the observation based on materials science is applied so that the total stress in BCC microstructures is additive in the form of the internal and effective stress components [18]. The unique feature of this constitutive relation [18] is an assumption that the strain hardening is rate and temperature sensitive. In the case of steels, when the strain rate $\dot{\varepsilon}$ increases a decrease of the rate of strain hardening is observed at the same time [18]. This is due to dislocation multiplication and thermal softening [18]. In the Rusinek-Klepaczko formulation [18], by similarity to the physical models, for example $[20,25-27,29,60,61]$, is assumed that the total stress is the sum of two components, the internal stress $\hat{\sigma}_{\mu}\left(\hat{\varepsilon}_{p}, \dot{\hat{\varepsilon}}_{p}, T\right)$ and the effective stress $\hat{\sigma}_{.}\left(\dot{\hat{\varepsilon}}_{p}, T\right)$. In extended of the RusinekKlepaczko model [19] a third stress component is added for defining viscous-drag stress at high strain rates as it was discussed in [62]:

$$
\hat{\sigma}\left(\hat{\varepsilon}_{p}, \dot{\hat{\varepsilon}}_{p}, T\right)=\frac{E(T)}{E_{0}}\left[\hat{\sigma}_{\mu}\left(\hat{\varepsilon}_{p}, \dot{\hat{\varepsilon}}_{p}, T\right)+\hat{\sigma}_{.}\left(\dot{\hat{\varepsilon}}_{p}, T\right)\right]+\hat{\sigma}_{d}\left(\dot{\hat{\varepsilon}}_{p}\right) .
$$

The term acting as multiplicative factor of the stresses addition $E(T) / E_{0}$ defines the Young's modulus evolution with temperature [63]:

$$
E(T)=E_{0}\left\{1-\frac{T}{T_{m}} \exp \left[\theta_{*}\left(1-\frac{T}{T_{m}}\right)\right]\right\} .
$$

The internal stress is determined by

$$
\hat{\sigma}_{\mu}\left(\hat{\varepsilon}_{p}, \dot{\hat{\varepsilon}}_{p}, T\right)=B\left(\dot{\hat{\varepsilon}}_{p}, T\right)\left(\varepsilon_{0}+\hat{\varepsilon}_{p}\right)^{n\left(\hat{\varepsilon}_{p}, T\right)} \text {. }
$$

The explicit formulations describing the modulus of plasticity and the strain hardening exponent are given:

$$
\begin{aligned}
& B\left(\dot{\hat{\varepsilon}}_{p}, T\right)=B_{0}\left(\left(\frac{T}{T_{m}}\right) \log \left(\frac{\dot{\varepsilon}_{\text {max }}}{\dot{\hat{\varepsilon}}_{p}}\right)\right)^{-v}, \\
& n\left(\dot{\hat{\varepsilon}}_{p}, T\right)=n_{0} \begin{cases}1-D_{2}\left(T / T_{m}\right) \log \left(\dot{\hat{\varepsilon}}_{p} / \dot{\varepsilon}_{\text {min }}\right), & 1-D_{2}\left(T / T_{m}\right) \log \left(\dot{\hat{\varepsilon}}_{p} / \dot{\varepsilon}_{\text {min }}\right) \geq 0 \\
0, & 1-D_{2}\left(T / T_{m}\right) \log \left(\dot{\hat{\varepsilon}}_{p} / \dot{\varepsilon}_{\text {min }}\right)<0\end{cases}
\end{aligned}
$$

Thus, based on experimental observations, [62], set the following relations

$$
\hat{\sigma}_{d}\left(\dot{\hat{\varepsilon}}_{p}\right)=\chi\left[1-\exp \left(-\alpha_{D} \dot{\hat{\varepsilon}}_{p}\right)\right] \text {. }
$$

The effective stress is obtained using Arrhenius relation which couples temperature with strain rate:

$$
\hat{\sigma}_{*}\left(\dot{\hat{\varepsilon}}_{p}, T\right)=\sigma_{*}^{0}\left\{\begin{array}{ll}
{\left[1-D_{1}\left(T / T_{m}\right) \log \left(\dot{\hat{\varepsilon}}_{\max } / \dot{\varepsilon}_{p}\right)\right]^{m .},} & 1-D_{2}\left(T / T_{m}\right) \log \left(\dot{\hat{\varepsilon}}_{\max } / \dot{\varepsilon}_{p}\right) \geq 0 \\
0, & 1-D_{2}\left(T / T_{m}\right) \log \left(\dot{\hat{\varepsilon}}_{\max } / \dot{\varepsilon}_{p}\right)<0
\end{array} .\right.
$$

All parameter designations of extended the RK model are presented in Table 4. As empirical CS model and the $\mathrm{JC}$ model, an explicit function predicting the strain rate dependence of plastic deformation is given by two parameters $\chi$ and $\alpha_{D}$. Let us apply the extended the RK model and the RP model to compression split Hopkinson bar tests of Al alloy ( $E=55 \mathrm{GPa} \sigma_{\mathrm{y}}=600 \mathrm{MPa}$ ). Parameters of the RP model equal to $\alpha=4.2, \tau=4.5$ $\mu \mathrm{s}, \beta=0.06$. Fig. 9 illustrates a comparison of prediction of stress-strain relationships by the RK model and the RP model at strain rate $0.01 \mathrm{~s}^{-1}, 1100 \mathrm{~s}^{-1}$ and $2200 \mathrm{~s}^{-1}$. The RK model begins modeling of plastic deformation with low yield stress unlike the RP model. In the case of yield drop, the same prediction of stress-strain dependence by the RK model was shown in the paper [64].

Let us now consider tension tests of B500A steel, performed in the paper [6]. By the RP model (Fig. 10a) and the $\mathrm{JC}$ model (Fig. 10b), stress-strain relationships at strain rates $0.001 \mathrm{~s}^{-1}$ and $1000 \mathrm{~s}^{-1}$ are plotted. Parameters of the RP model equal to $\tau=1.48 \mu \mathrm{s}, \alpha=1, \beta=0.032$. Similarly by plastic deformation of 

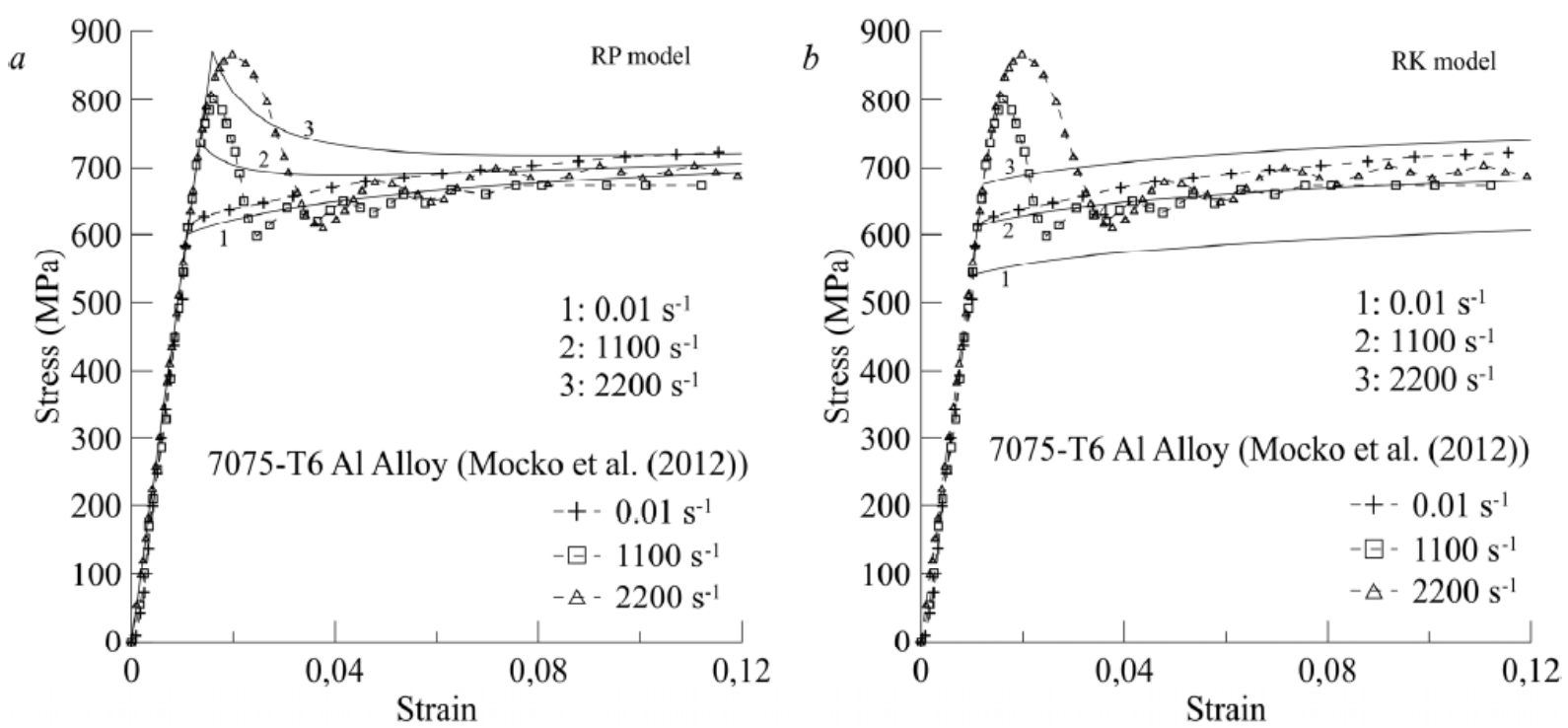

Fig. 9. Stress-strain relationships of aluminum alloy 7075-T6 (data from [48]), plotted by RP model (a) and RK model (b).
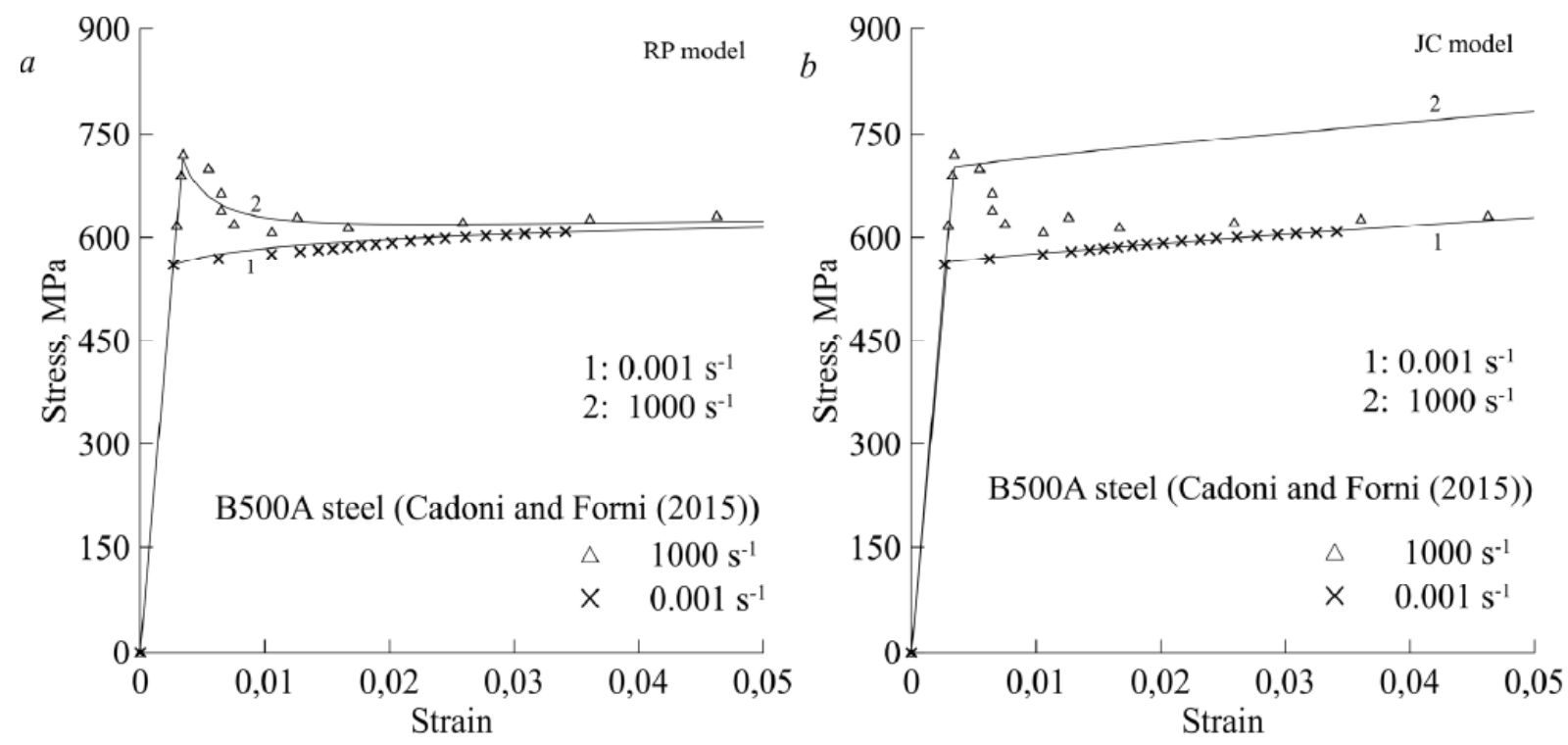

Fig. 10. Stress-strain dependences of B500A steel [6] on strain rates $0.001 \mathrm{~s}^{-1}$ and $1000 \mathrm{~s}^{-1}$, predicted by the RP model (a) and the JC model (b).

TRIP 800 steel (Fig. 5) and $590 \mathrm{MPa}$ grade steel (Fig. 6), the yield drop phenomenon at strain rate $1000 \mathrm{~s}^{-1}$ and monotonic transition from elastic to plastic deformation at $0.001 \mathrm{~s}^{-1}$ are observed. The $\mathrm{JC}$ model can predict only typical stress-strain relations where the yield stress can change while the relation keeps to being monotonic. However, the phenomenological RP model is explained and predicted the dynamic effects of plasticity using a fixed set of parameters.

\section{CONCLUSIONS}

Predictions of strain rate sensitivity of the material by different dynamic models of plastic deformation are reviewed. The CS model can yield results that coincide with the results of the proposed incubation time criterion when determining the yield strength as a function of the strain rate. The advantage of calculating the yield strength by the incubation time criterion is a limited set of material parameters that do not require further modifications in conditions of high strain rates. Besides empirical models based on the $\mathrm{JC}$ model and the phenomenological extended RK model we considered the relaxation model plasticity as a generalized model of plastic deformation based on the structural-temporal approach. It is shown that the relaxation model predicts the yield drop effect during the high-rate deformation of TRIP800 steel, $590 \mathrm{MPa}$ grade steel and an ab- 
Table 4. Parameters of extended RusinekKlepaczko model.

Mechanical properties of material

$\begin{array}{ll}E_{0} & \text { Young's modulus at } T=0 \mathrm{~K} \\ T_{\mathrm{m}} & \text { Melting temperature }\end{array}$

Material constants of hardening

\begin{tabular}{ll}
\hline$B_{0}, D_{2}$, & Material constants \\
$n_{0}$ & Strain hardening exponent at $T=0 \mathrm{~K}$ \\
$\theta$ & The characteristic homologous tem- \\
& perature \\
$v$ & Temperature dependence
\end{tabular}

Material constants of temperature dependence

\begin{tabular}{ll}
\hline$\sigma_{*}^{0}, D_{1}$ & Material constants \\
$\mathrm{m}_{*}$ & $\begin{array}{l}\text { Constant allowing to define the strain } \\
\text { rate-temperature dependence }\end{array}$
\end{tabular}

Strain rate parameters

\begin{tabular}{ll}
\hline & Material constant \\
$\alpha_{D}$ & an effective damping coefficient affect- \\
& ing the dislocation motion [19]
\end{tabular}

Parameter of model

\begin{tabular}{ll}
\hline$\dot{\varepsilon}_{\text {min }}$ & the lower limit of the model \\
$\dot{\varepsilon}_{\max }$ & $\begin{array}{l}\text { the maximum strain rate accepted for } \\
\text { a particular material }\end{array}$ \\
\hline
\end{tabular}

sence of such effect for a wide range of strain rates for the 2.3Ni-1.3Cr steel. The relaxation model gave better correspondence of stress-strain relations in conditions of high strain rates with experimental data compared to the improved JC models. This approach can describe the anomalous behaviour of plastic deformation, such as the yield drop. It is shown that improved JC model and RK model don't take into account yield drop phenomenon, observed in soft steels and 7075-T6 aluminium alloy. Thus, the relaxation model of plasticity is an efficient and convenient tool for calculations of some of the principal dynamic plasticity effects occurring within a wide range of strain rates.

\section{ACKNOWLEDGEMENTS}

The study was supported by the Russian Science Foundation, project no. 17-71-10061.

\section{REFERENCES}

[1] A. Uenishi and C. Teodosiu // Int. J. Plasticity 20 (2004) 915.

[2] S. Takagi, Y. Tokita, K. Sato and T. Shimizu, SAE Technical Paper 2005-01-0494 (2005).

[3] P. Larour, A. Rusinek, J.R. Klepaczko and W. Bleck // Steel Res. Int. 78 (2007) 348.

[4] E. Cadoni, F.D. Aiuto and C. Albertini // DYMAT 2009 (2009) 135.

[5] X. Sun, A. Soulami, K.S. Choi, O. Guzman and W. Chen // Mater. Sci. Engng. A 541 (2012) 1.

[6] E. Cadoni and D. Forni // EPJ Web Conf. 94 (2015) 01004.

[7] A.S. Khan, Y.S. Suh and R. Kazmi // Int. J. Plast. 20 (2004) 2233.

[8] Y.C. Lin, X.-M. Chen and G. Liu // Mater. Sci. Engng. A 527 (2010) 6980.

[9] Y.P. Wang, C.J. Han, C. Wang and S.K. Li // J. Mater. Sci. 46 (2011) 2922.

[10] L. Gambirasio and E. Rizzi // Comp. Mater. Sci. 113 (2016) 231.

[11] G.R. Johnson and W.H. Cook // Eng. Fract. Mech. 21 (1985) 31.

[12] T.J. Holmquist and G.R. Johnson // J. Phys. IV 1 (1991) 299.

[13] G.T. Camacho and M. Ortiz // Comput. Method. Appl. M. 142 (1997) 269.

[14] W.K. Rule and S.E. Jones // Int. J. Impact Eng. 21 (1998) 609.

[15] A. Khan and R. Liang // Int. J. Plasticity 15 (1999) 1089.

[16] H. Couque, R. Boulanger and F. Bornet // J. Phys. IV 134 (2006) 87.

[17] A.K. Maheshwari, K.K. Pathak, N. Ramakrishnan and S.P. Narayan // J. Mater. Sci. 45 (2010) 859.

[18] A. Rusinek and J.R. Klepaczko // Int. J. Plasticity 17 (2001) 87.

[19] A. Rusinek and J.A. Rodriguez-Martinez // Materials and Design 30 (2009) 4377.

[20] A. Seeger, Dislocations and Mechanical Properties of Crystals (Wiley, New York, 1957).

[21] H. Conrad // J. Metals 16 (1964) 582.

[22] J.J. Gilman // Appl. Mech. Rev. 21 (1968) 767.

[23] G.B. Gibbs // Mater. Sci. Engng. 4 (1969) 313.

[24] U.F. Kocks, A.S. Argon and M.F. Ashby, In: Progress in Materials Science, vol. 19.

(Pergamon Press: New York, 1975), p. 19. 
[25] U.F. Kocks // J. Eng. Mater. Technol. 98 (1976) 76.

[26] J.R. Klepaczko // Mater. Sci. Engng. 18 (1975) 121.

[27] J.R. Klepaczko and G.C.Y. Chiem // J. Mech. Phys. Solids 34 (1986) 29.

[28] J.R. Klepaczko // J. Mech. Working Technology 15 (1987) 143.

[29] F.J. Zerilli and R.W. Armstrong // J. Appl. Phys. 61 (1987) 1816.

[30] P.S. Follansbee and U.F. Kocks // Acta Metall. 36 (1988) 81.

[31] P.S. Follansbee and G.T. Gray III // Mater. Sci. Eng. A 138 (1991) 23.

[32] A. Molinari and G. Ravichandran // Mech. Mater. 37 (2005) 737.

[33] A.A. Gruzdkov and Yu.V. Petrov // Dokl. Phys. 44 (1999) 114.

[34] A.A. Gruzdkov, Yu.V. Petrov and V.I. Smirnov // Phys. Solid State 44 (2002) 2080.

[35] Yu.V. Petrov and Y.V. Sitnikova // Tech. Phys. 50 (2005) 1034.

[36] Yu.V. Petrov, A.A. Gruzdkov and E.V. Sitnikova // Dokl. Phys. 52 (2007) 691.

[37] A.A. Gruzdkov, E.V. Sitnikova, N.F. Morozov and Y.V. Petrov // Math. Mech. Solids 14 (2009) 72.

[38] Y.V. Petrov // Mech. Solids 42 (2007) 692.

[39] Y.V. Petrov and A.A. Utkin // Sov. Mater. Sci. 25 (1989) 153.

[40] A.N. Berezkin, S.I. Krivosheev, Yu.V. Petrov and A.A. Utkin // Dokl. Phys. 45 (2000) 617.

[41] V.A. Bratov, A.A. Gruzdkov, S.I. Krivosheev and Yu.V. Petrov // Dokl. Phys. 49 (2004) 338.

[42] Yu.V. Petrov and E.N. Borodin // Phys. Solid State 57 (2015) 353.

[43] N. Selyutina, E.N. Borodin, Y. Petrov and A.E. Mayer // Int. J. Plasticity 82 (2016) 97.

[44] N.S. Selyutina and Yu.V. Petrov // Dokl. Phys. 62 (2017) 102.

[45] N.S. Selyutina, E.N. Borodin and Yu. V. Petrov // Phys. Solid State 60 (2018) 1813.

[46] G.R. Cowper and P.S. Symonds, Report no. 28 (Div. Appl. Math., Brown Univ., Providence, RI, USA, 1957).
[47] E. Cadoni, N.K. Singh, D. Forni, M.K. Singha and N.K. Gupta // Eur. Phys. J. Spec. Top. 225 (2016) 409.

[48] W. Mocko, J.A. Rodriguez-Martinez, Z.L. Kowalewski and A. Rusinek // Strain 48 (2012) 498.

[49] N.S. Selyutina and Yu.V. Petrov // Phys. Solid State 60 (2018) 244.

[50] L. Schwer, In: Proceedings of the 6th European LS-DYNA Users' Conference (Gothenburg, 2007).

[51] G.I. Mylonas and G.N. Labeas // Exp. Techniques 38 (2014) 26.

[52] D.-N. Zhang, Q.Q. Shangguan, C.-J. Xie and F. Liu // J. Alloy. Compd. 619 (2014) 186.

[53] I.N. Borodin and Y.V. Petrov // Mech. Solids 49 (2014) 635.

[54] P.R. Guduru, P.R. Singh, G. Ravichandran and A.J. Rosakis // J. Mech. Phys. Solids. 46 (1998) 1997.

[55] W.-H. Liu, Z.-T. He, Y.-Q. Chen and S.-W. Tang // Trans. Nonferrous Met. Soc. China 24 (2014) 2179.

[56] W.J. Kang, S.S. Cho, H. Huh and D.T. Chung // Int. J. Vehicle Design 21 (1999) 424.

[57] B.J. Tuazon, K.-O. Bae, S.-H. Lee and H.-S. Shin // J. Mech. Sci. Technol. 28 (2014) 3561.

[58] A.S. Khan, Y.S. Sun and R. Kazmi // Int. J. Plasticity 20 (2004) 2233-2248.

[59] J. Che, T. Zhou, Z. Liang, J. Wu and X. Wang // J. Braz. Soc. Mech. Sci. 40 (2018) 253.

[60] J.R. Klepaczko, In: Proc. Int. Conf. on High Energy Rate Fabrication (New York, 1984), p. 45.

[61] M.A. Meyers, D.J. Benson, O. Vohringer, B.K. Kad, Q. Xue and H. Fu // Mater. Sci. Engng. A 322 (2002) 194.

[62] R. Kapoor and S. Nemat-Nasser // Metall. Mater. Trans. 31A (1999) 815.

[63] J.R. Klepaczko, In: Impact: effects of fast transient loadings (Rotterdam, 1998), p. 3.

[64]A. Rusinek, J.A. Rodríguez-Martínez, J.R. Klepaczko and R.B. Pecherski // Materials and Design 30 (2009) 1748. 